\title{
Pneumothorax in SARS-CoV-2 Affected Patients
}

\author{
${ }^{1}$ Department of Medicine, Justice K.S. Hegde Hospital, Deralakatte, \\ Mangalore, Karnataka, India \\ ${ }^{2}$ Department of Pulmonary Medicine, Justice K.S. Hegde Hospital, \\ Deralakatte, Mangalore, Karnataka, India \\ ${ }^{3}$ Department of Pulmonary Medicine, Justice K.S. Hegde Hospital, \\ Deralakatte, Mangalore, Karnataka, India
}

Srivatsa R. ${ }^{1} \quad$ Giridhar Belur Hosmane ${ }^{2} \quad$ Rajesh Venkataram³ ${ }^{3}$ Nandakishore Baikunje ${ }^{3, \odot}$

Address for correspondence Srivatsa R., MBBS, MD, Department of Medicine, K.S. Hegde Medical College, Deralakatte, Mangalore 575022, Karnataka, India (e-mail: srivatsa11.r@gmail.com).

\begin{abstract}
Keywords

- pneumothorax

- SARS-CoV-2

- mechanical ventilation

- complications

Severe acute respiratory syndrome coronavirus 2 (SARS-CoV-2) infection has various known complications in its natural course of the illness. Pneumothorax is one of the emerging complications in coronavirus disease 2019 (COVID-19)-positive patients, not initiated on invasive mechanical ventilation. Case studies and autopsy reports across the globe suggest alveolar pathology to be responsible for the outcome.

COVID-19 infection in humans caused by the SARS-CoV-2 virus has various known complications in its natural course of the illness. Pneumothorax is one of the emerging complications in COVID-19-positive patients. Prior identification and anticipation of such life-threatening complications aid in the treatment of COVID-19-positive patients.
\end{abstract}

\section{Introduction}

Coronavirus disease 2019 (COVID-19) infection is caused by a novel coronavirus, severe acute respiratory syndrome coronavirus 2 (SARS-CoV-2), that has infected millions of individuals across the world. This disease is most frequently manifested as a pulmonary disease, although recent reports have described it to be a systemic disease. ${ }^{1}$ The disease most frequently manifests as a viral pharyngitis or viral pneumonia characterized by fever, cough, and breathlessness or can cause life-threatening pulmonary complications like acute respiratory distress syndrome (ARDS). ${ }^{2}$ Extrapulmonary manifestations of COVID-19 like pleurisy, pleural effusion, lymphadenopathy, and pneumothorax are uncommon presentations of COVID-19 infection. Apart from these respiratory complications, secondary complications like acute kidney injury or cardiac injury were common.

Spontaneous pneumothorax is the accumulation of extrapulmonary air within the chest, not caused by trauma or iatrogenic factors. Primary spontaneous pneumothorax occurs without any clinically apparent lung disease, whereas secondary pneumothorax occurs as a complication of a preexisting lung disease. Pneumothorax seen in chronic tobacco users bronchiectasis and emphysematous lung is commonly heard of; however, it is an uncommon complication of COVID pneumonia

Herein we report two cases of SARS-CoV-2 disease that developed pneumothorax on noninvasive ventilator (NIV) support.

\section{Case Presentation}

\section{Case 1}

A 42-year-old male patient, known case of systemic hypertension and chronic kidney disease for the last 3 years on maintenance hemodialysis-twice weekly-was admitted with 3-day history of cough and breathlessness. At the time of admission, his oxygen saturation was $90 \%$ on room air. He was started on supplementary oxygen with facemask at $6 \mathrm{~L} / \mathrm{min}$ and oxygen saturation improved to $96 \%$. Chest X-ray taken at the time of admission showed nonhomogeneous opacity in the right upper, middle, and lower lobes and left published online

August 18, 2021
DOI https://doi.org/

$10.1055 / \mathrm{s}-0041-1734320$ ISSN 2582-4287 (c) 2021. Nitte (Deemed to be University).

Nitte University. This is an open access article published by Thieme under the terms of the Creative Commons Attribution-NonDerivative-NonCommercial-License, permitting copying and reproduction so long as the original work is given appropriate credit. Contents may not be used for commercial purposes, or adapted, remixed, transformed or built upon. (https://creativecommons.org/licenses/by-nc-nd/4.0/). Thieme Medical and Scientific Publishers Pvt. Ltd. A-12, 2nd Floor, Sector 2, Noida-201301 UP, India 
middle lobe. COVID-19 reverse transcription polymerase chain reaction (RT-PCR) test was found to be positive. Patient was admitted in COVID-19 intensive care unit (ICU) and treatment was started based on institutions COVID-19 protocol.

With increasing oxygen demands, patient was ventilated with nonrebreather mask (NRBM) at $12 \mathrm{~L} / \mathrm{min}$ on day 2 of admission and gradually increased to $15 \mathrm{~L} / \mathrm{min}$ on day 3 of admission. Chest X-ray showed worsening ARDS and blood investigations showed worsening sepsis with a rise in inflammatory markers. His oxygen requirement continued to increase and was initiated on NIV (fraction of inspired oxygen $\left[\mathrm{FiO}_{2}\right]-100 \%$ and positive end-expiratory pressure [PEEP]-6) on day 5 of admission. He was closely monitored with arterial blood gas (ABG) to maintain partial pressure of oxygen $\left(\mathrm{PaO}_{2}\right)$ $>60$ and was hemodialyzed thrice during the hospital stay to treat uremia. NIV settings were adjusted accordingly by lowering FiO2 to prevent oxygen-related free radical damage and maintain $\mathrm{PaO} 2>60$. On day 7 of admission, patient was maintaining saturation of 85 to $90 \%$ on NIV (PEEP-6, $\mathrm{FiO}_{2}-$ $90 \%$, tidal volume [TV]-300-350). Examination revealed subcutaneous emphysema in the neck (right > left). Findings were confirmed with a chest X-ray that showed presence of subcutaneous emphysema associated with pneumomediastinum (-Fig. 1). Patient was hemodynamically stable; hence, he was symptomatically managed. Chest X-ray on day 8 of admission (day 3 of NIV) showed large pneumothorax on the right side and a small pneumothorax on the left side (-Fig. 2). Intercostal drainage tubes were placed into both the pleural cavities. Post-tube insertion X-rays showed adequate re-expansion of the lungs; however, he had a complicated clinical course in the hospital and the patient succumbed to his illness in the second week of the hospitalization.

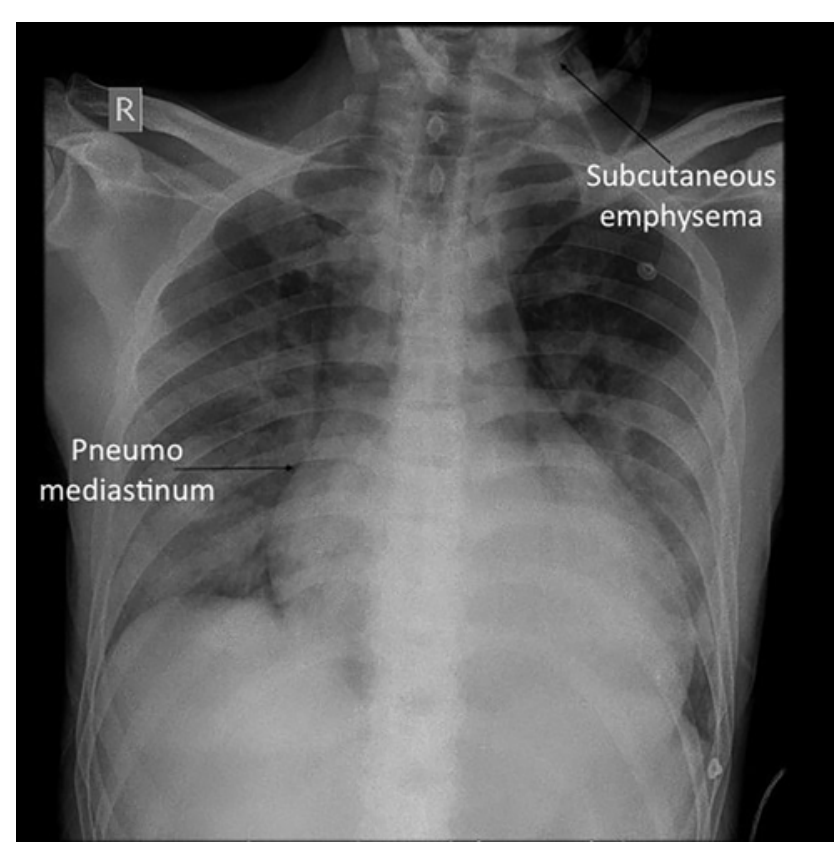

Fig. 1 Chest radiograph of case 1 denoting right subcutaneous emphysema and pneumomediastinum with intercostal drainage in situ.

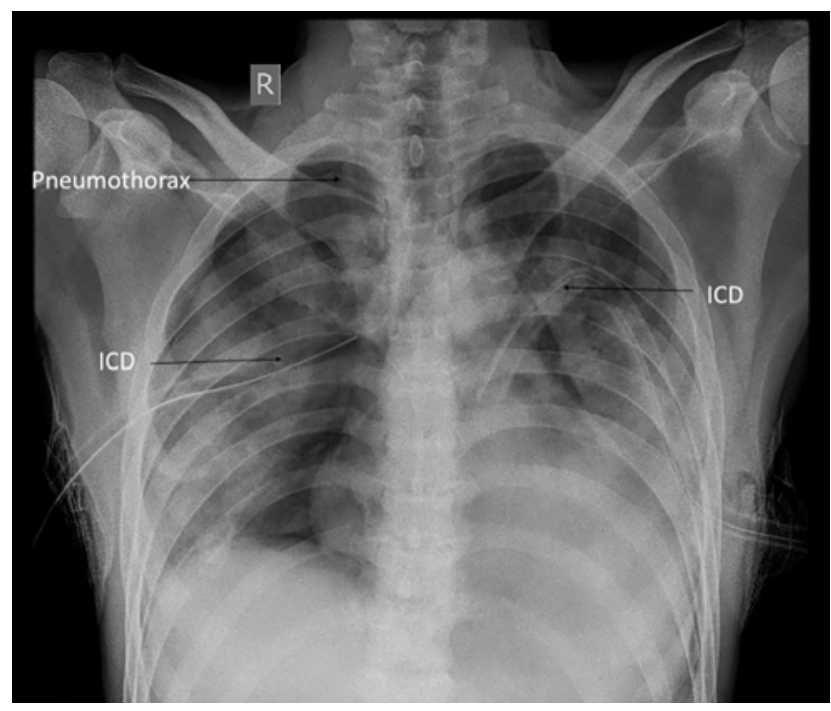

Fig. 2 Chest radiograph of case 1 denoting bilateral pneumothorax with intercostal drainage in situ (ICD).

\section{Case 2}

A 63-year-old male, nonsmoker with no known comorbidities, presented to the hospital with a 3-day history of fever, cough, and breathlessness. On arrival, his saturation was 69\% on room air, which improved to $95 \%$ on $15 \mathrm{~L} / \mathrm{min}$ oxygen with a facemask. Chest radiograph on admission showed nonhomogeneous opacities in the left middle and lower zones and right lower zone, suggestive of viral pneumonia. RT-PCR of nasopharyngeal swab for COVID-19 was positive. He was started on treatment in ICU as per institutional COVID-19 protocol. His ABG revealed persistent hypoxemia ( $\mathrm{PaO} 2<60)$; hence, oxygen delivery system was changed to NRBM which started at $10 \mathrm{~L} / \mathrm{min}$ and increased to $15 \mathrm{~L} / \mathrm{min}$ to achieve saturation of $95 \%$. On day 5 of admission, patient was found to be desaturating with NRBM; hence, he was initiated on NIV (PEEP-5, $\mathrm{FiO}_{2}-100 \%, \mathrm{TV}-400-450 \mathrm{~mL}$ ). Ventilator settings were gradually adjusted to maintain $\mathrm{PaO} 2>60 \%$. The following day, settings were changed: NIV (PEEP-7, $\mathrm{FiO}_{2}-80 \%$, TV-400-450 mL). Blood parameters showed leukocytosis. Liver and kidneys appeared normal in their respective biochemical parameters. On day 7 of illness, NIV was set at PEEP-8, $\mathrm{FiO}_{2}-60 \%, \mathrm{TV}-350-400 \mathrm{~mL}$. His hemodynamic parameters were stable.

He developed spontaneous atrial fibrillation on day 9 of hospitalization. New onset atrial fibrillation was reverted with intravenous medication after two-dimensional echocardiogram showed no signs of ischemia. Patient was still tachypneic and tachycardic with normal blood pressure. To maintain a $\mathrm{PaO}_{2}>60$, NIV (day 4 on NIV) settings were maintained at PEEP-10, $\mathrm{FiO}_{2}-60, \mathrm{TV}-350-400 \mathrm{~mL}$. Chest X-ray was done that showed pneumothorax on right side (-Fig. 3 ). Findings were confirmed with ultrasound thorax, which showed absence of lung sliding on right side. Implantable cardioverter defibrillator was immediately inserted to decompress the pneumothorax (-Fig. 4). He succumbed to the illness the following day. 


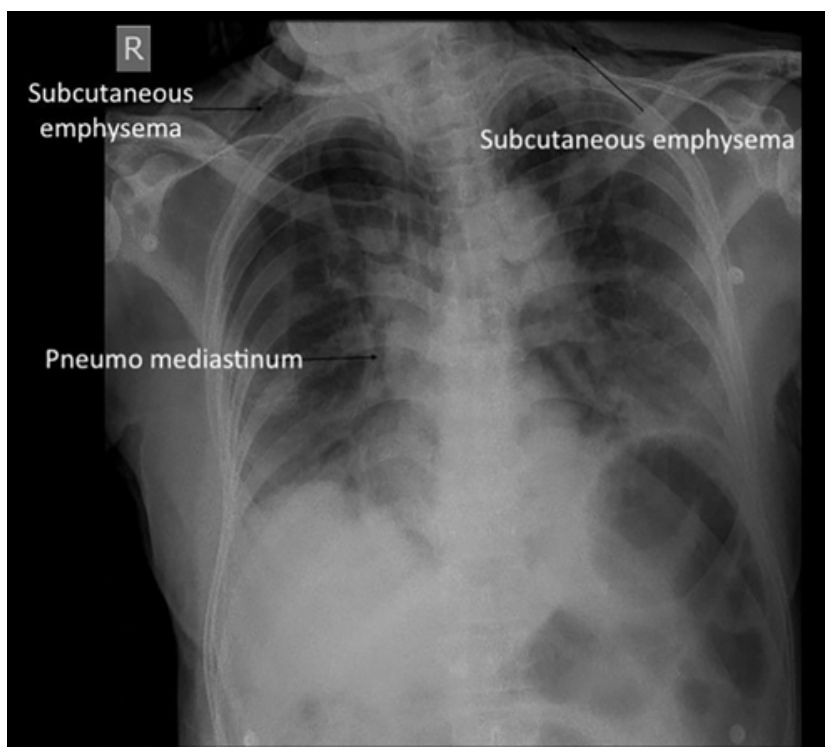

Fig. 3 Chest radiograph of case 2 denoting right subcutaneous emphysema and pneumomediastinum.

\section{Discussion}

COVID-19 is an atypical pneumonia caused by SARS-CoV-2 virus. The virus has infected more than 50 million people worldwide, spread via respiratory droplets and airborne factors. Respiratory system was the primary organ involved in all the COVID-19-infected patients. Studies have shown to involve both the upper and lower respiratory tracts in the form of interstitial pneumonia, acute respiratory distress, bronchiolitis, vasculitis, and pulmonary embolism. Pleural involvement in this disease is found to be less frequent and is reported in very few instances in patients not on invasive mechanical ventilation, across the globe. ${ }^{4,5}$

In one such instance, two COVID-19-positive patients admitted in our hospital with respiratory distress developed pneumothorax. It is imperative to note that these patients had no preexisting lung abnormalities. Although we do not have any pre-COVID-19 lung imaging to confirm the absence of lung injury, we did not have any reason to suspect it based on the history. They presented early in the disease course with need for supplemental oxygen. They were admitted in the ICU and managed according to institutions COVID-19 protocol. They were initiated early on NIV to provide positive pressure ventilation. Effective ventilation strategy for ARDS (low TV and high PEEP) was followed despite which the two patients developed pneumothorax during hospitalization.

It is also important to note that both our patients had severe COVID-19 pneumonia and their health deteriorated gradually but progressively, despite adequate treatment given. It appears that both the patients developed pneumothorax in the second week of the illness, when patients were administered oxygen through NIV. Prior to the development of pneumothorax, it was observed that both the patients had developed subcutaneous emphysema. Hence, paying close attention to extraparenchymal structures in the chest X-ray

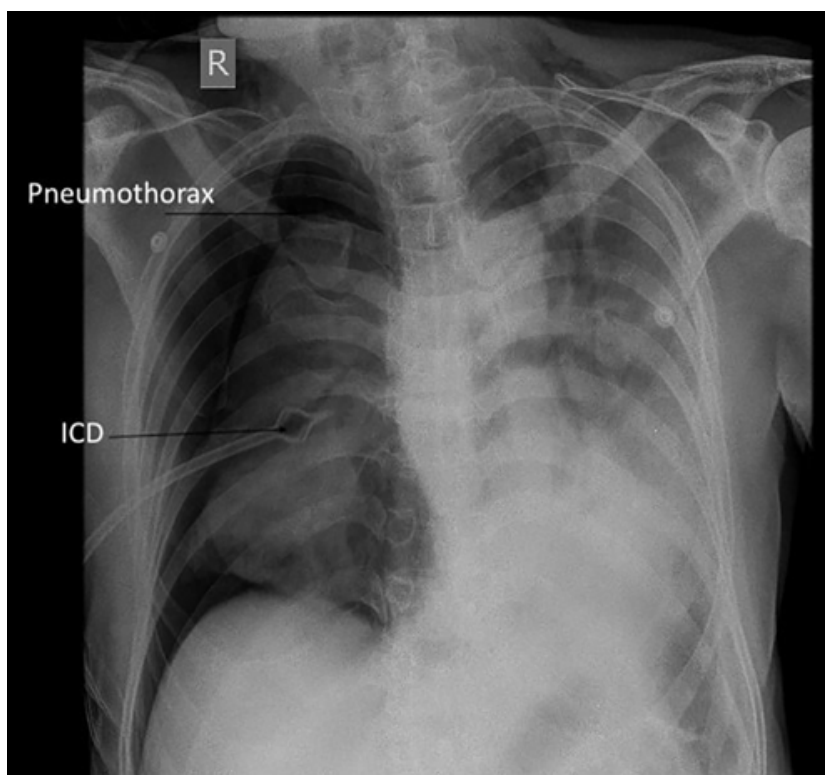

Fig. 4 Chest radiograph of case 2 denoting right pneumothorax with intercostal drainage (ICD) in situ.

like subcutaneous tissue mediastinum helps to detect early features of air leak, that is, subcutaneous emphysema and/or spontaneous pneumomediastinum. Inadequate deliveries of the TV and high peak pressures on the ventilators are other early signs of development of pneumothorax.

Pneumothorax is a serious complication, if not managed immediately. Early decompression prevents morbidity and mortality. Pneumothorax as a complication of viral illness was noticed in the previous outbreaks of SARS-CoV-1, Middle East respiratory syndrome (MERS), and human immunodeficiency virus with no predisposing lung disease. It was also indicative of poor prognosis and high short-term mortality. ${ }^{6}$ SARS-CoV-2 virus is known to cause COVID-19 pneumonia and ARDS as its commonest complications.

While causality cannot be established through a case series between COVID-19 and pneumothorax, our series adds to the many published articles on COVID-19 and pneumothorax, adding to the weight of this being an associated complication of COVID-19 rather than being merely coincidental/ventilator-related barotrauma. ${ }^{7}$ Explaining the relationship between pneumothorax and COVID-19 is a task with multiple possibilities.

In a case series of six patients with COVID-19 pneumonia and pneumothorax, it was seen that high peripheral leucocyte count (neutrophil count, neutrophil-lymphocyte ratio) was postulated to depict a greater extent of lung injury that raises the risk of pneumothorax. ${ }^{8}$ They had rising inflammatory markers, a high $\mathrm{C}$-reactive protein, and neutrophil percentage at the time of development of pneumothorax. Similar data was noted in our case series ( - Table $\mathbf{1}$ ).

Radiologically, patients with SARS-CoV-1 and MERS are known to show ground glass opacities evolving into consolidative changes and finally into fibrosis. Similar changes including diffuse alveolar injury, pneumatoceles due to the virus, and exudates are known to occur in COVID-19 pneumonia that are thought to contribute to pneumothorax. These lung 
Table 1 Blood investigations of SARS-CoV-2-affected cases showing worsening titers of inflammatory markers from the time of admission to the onset of pneumothorax

\begin{tabular}{|l|l|l|l|l|}
\hline & \multicolumn{2}{|c|}{ Case 1 } & \multicolumn{2}{c|}{ Case 2 } \\
\hline & Admission & $\begin{array}{l}\text { On the day of detection } \\
\text { of pneumothorax-(D8 } \\
\text { since admission) }\end{array}$ & Admission & $\begin{array}{l}\text { On the day of detection of } \\
\text { pneumothorax-(D9 since } \\
\text { admission) }\end{array}$ \\
\hline Total WBC count $(/ \mathrm{dL})$ & 7,500 & 11,800 & 5,800 & 15,800 \\
\hline N/L ratio & 15.5 & 18.8 & 8.9 & 32 \\
\hline Albumin $(\mathrm{g} / \mathrm{dL})$ & 3.75 & 3.61 & 3.31 & 4.29 \\
\hline Serum ferritin $(\mathrm{ng} / \mathrm{mL})$ & 6,384 & 8,896 & 1,831 & 1,485 \\
\hline CRP $(\mathrm{mg} / \mathrm{L})$ & 301.99 & 359.7 & 101.99 & 17.11 \\
\hline
\end{tabular}

Abbreviations: CRP, C-reactive protein; N/L, neutrophil/lymphocyte; SARS-CoV-2, severe acute respiratory syndrome coronavirus 2; WBC, white blood cell.

changes lead to overdistention of alveoli even with minimal positive pressure (NIV) leading to pneumothorax. ${ }^{9}$ With limited evidence for disease-modifying therapy, management of patients with severe disease has relied on respiratory support. When supplemental oxygen with open system is insufficient, advanced respiratory support systems like NIV and invasive mechanical ventilation are required. Patients on mechanical ventilators are ventilated with positive pressure as opposed to the normal physiological process; thus, complications like barotrauma and volutrauma are common. However, the incidence of barotrauma in patients receiving noninvasive mechanical ventilation is much lower when compared with patients receiving invasive mechanical ventilation. ${ }^{10,11}$

Development of pneumothorax in SARS-CoV-2 infection has been thought of as a poor prognostic marker. ${ }^{12}$ Both the patients, who developed pneumothorax, succumbed to death in the following days, despite adequate emergent measures taken to decompress the pneumothorax.

\section{Conclusion}

Pneumothorax occurs in COVID-19 pneumonia patients, despite the use of lung protective ventilatory approach, making barotrauma a less likely cause for it. Close attention must be paid to the extraparenchymal structures in chest X-ray to detect the features of air leak early.

\section{Conflict of Interest}

None declared.

\section{References}

1 Yuki K, Fujiogi M, Koutsogiannaki S. COVID-19 pathophysiology: a review. Clin Immunol 2020;215:10842710.1016/j. clim.2020.108427
2 Huang C, Wang Y, Li X, et al. Clinical features of patients infected with 2019 novel coronavirus in Wuhan, China. Lancet 2020;395(10223):497-506

3 Zaim S, Chong JH, Sankaranarayanan V, Harky A. COVID-19 and multiorgan response. Curr Probl Cardiol 2020;45(8):100618

4 Lang M, Som A, Carey D, et al. Pulmonary vascular manifestations of COVID-19 Pneumonia: radiology. Cardiothoracic Imag 2020;2(3)

5 Xu Z, Shi L, Wang Y, et al. Pathological findings of COVID-19 associated with acute respiratory distress syndrome. Lancet Respir Med 2020;8(4):420-422

6 Terzi E, Zarogoulidis K, Kougioumtzi I, et al. Human immunodeficiency virus infection and pneumothorax. J Thorac Dis 2014;6(Suppl 4):S377-S382

7 Sun R, Liu H, Wang X. Mediastinal emphysema, giant bulla, and pneumothorax developed during the course of COVID-19 pneumonia. Korean J Radiol 2020;21(5):541-544

8 Martinelli AW, Ingle T, Newman J, et al. COVID-19 and pneumothorax: a multicentre retrospective case series. Eur Respir J 2020;56(5):2002697

9 Sihoe AD, Wong RH, Lee AT, et al. Severe acute respiratory syndrome complicated by spontaneous pneumothorax. Chest 2004;125(6):2345-2351

10 Zantah M, Dominguez Castillo E, Townsend R, Dikengil F, Criner GJ. Pneumothorax in COVID-19 disease- incidence and clinical characteristics. Respir Res 2020;21(1):236

11 López Vega JM, Parra Gordo ML, Diez Tascón A, Ossaba Vélez S. Pneumomediastinum and spontaneous pneumothorax as an extrapulmonary complication of COVID-19 disease. Emerg Radiol 2020;27(6):727-730

12 Carron M, Freo U, BaHammam AS, et al. Complications of non-invasive ventilation techniques: a comprehensive qualitative review of randomized trials. $\mathrm{Br} \mathrm{J}$ Anaesth 2013;110(6):896-914 